\title{
Variable period change of femtosecond written fiber Bragg gratings with a deformed wavefront
}

\author{
Christian Voigtländer ${ }^{1}$, Ria G. Krämer ${ }^{1}$, Jens U. Thomas ${ }^{1}$, \\ Daniel Richter ${ }^{1}$, Andreas Tünnermann ${ }^{1,2}$, and Stefan Nolte ${ }^{1,2}$ \\ ${ }^{1}$ Institute of Applied Physics, Abbe Center of Photonics, Friedrich-Schiller-University Jena, \\ Max-Wien-Platz 1, D-07743 Jena, Germany. \\ ${ }^{2}$ Fraunhofer Institute for Applied Optics and Precision Engineering, \\ Albert-Einstein-Strasse 7, D-07745 Jena, Germany \\ Authore-mail address: ch.voigtlaender@uni-jena.de
}

\begin{abstract}
We report on the inscription of fiber Bragg gratings using ultrashort laser pulses and the phase mask technique. By deforming the wavefront of the inscription laser a tuned reflection wavelength as well as broadband chirped gratings could be realized. A theoretical analysis and experimental results will be discussed.
\end{abstract}

\section{Introduction}

Fiber Bragg gratings (FBGs) are standard integrated optical components today. Within the last decade the interest in all-integrated fiber lasers has been growing. Here FBGs can be used as cavity mirrors. However, conventional UVinscription techniques reach their limits as they require photosensitive fibers. This drawback can be overcome by using femtosecond laser pulses for the inscription [1]. Thus, monolithic fiber laser concepts without splicing can be realized. For reproducible inscription of FBGs we employed the phase mask technique.

However, for various applications broadband gratings are required. Here we present a method for generating shifted and broadband FBGs by shaping the interference pattern of a phase mask using curved wavefronts of the inscribing beam.

\section{Experiments}
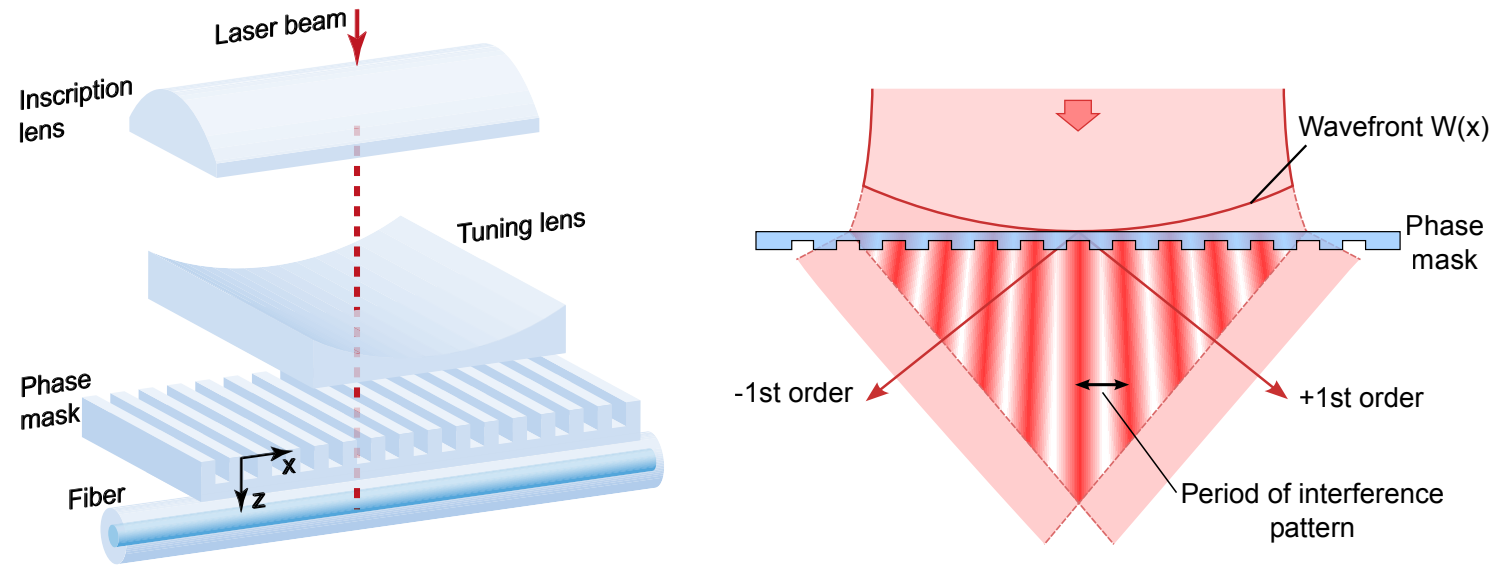

Fig. 1. Left side: Illustration of the inscription setup for tuning the grating period. Right side: Scheme of a deformed wavefront propagating through the phase mask with the resulting interference pattern. 
A scheme of the inscription technique is shown on the left side of Fig. (1). The femtosecond laser beam from a commercial regenerative amplified Ti:Sapphire system (CPA 2110, Clark-MXR) is focused by a 20 mm cylindrical lens through a phase mask into the core of a standard single mode fiber. [2]. An interference pattern between the -1st und +1 st diffraction order is generated underneath the phase mask. By using a plane wavefront and a phase mask with constant period the pattern will have constant period (half of that of the phase mask) as well. With a curved wavefront the incident angle to the mask changes along the beam cross section leading to a change of the period of the interference pattern.

For the experimental realization we have integrated a tuning lens between the focusing lens and the phase mask [3]. The tuning lens changes the wavefront along the fiber axis. By applying different curvature radii the period can be shifted to different wavelengths. We could show a tuning range of more than $300 \mathrm{~nm}$ with different tuning lenses and the same phase mask (see Fig. (2) left side). These results could be achieved just by a defocusing wavefront. Additionally the period of the FBG can be chirped by using higher order aberrations than the defocus term. These aberrations can be simply realized by tilting the tuning lens and passing it off-axis. Furthermore, the period changes with increasing distance from the phase mask. Thus, we could achieve a broadband reflection of $6 \mathrm{~nm}$ by using higher order aberrations and also tilting the fiber underneath the phase mask (see Fig. (2) right side).

In the future, defined wavefront curvatures imprinted by a spatial light modulator or adaptive mirror will be used.
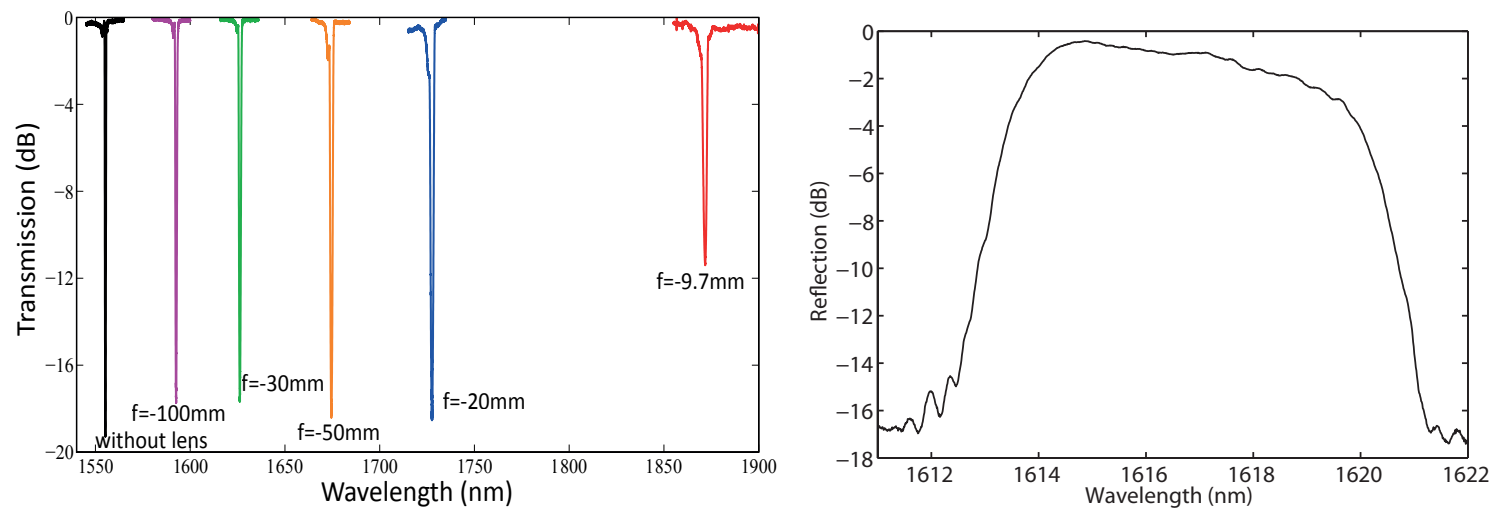

Fig. 2. Left side: Spectra of FBGs with different tuned grating periods due to different tuning lenses. Right side: Spectrum of a chirped FBG inscribed with a deformed wavefront.

\section{References}

1. J. Thomas, C. Voigtländer, R.G. Becker, D. Richter, A. Tünnermann, and S. Nolte, ’Femtosecond pulse written fiber gratings: a new avenue to integrated fiber technology,' Laser Photonics Rev. 6, no 6, pp. 709-723 (2012).

2. C. Voigtländer, R. G. Becker, J. Thomas, D. Richter, A. Singh, A. Tünnermann, and S. Nolte, "Chirped fiber Bragg gratings written with ultrashort pulses and a tunable phase mask," Opt. Mater. Express 1(4), pp. 633-642 (2011).

3. J. D. Prohaska, E. Snitzer, S. Rishton, and V. Boegli, ”Magnification of mask fabricated fibre Bragg gratings," Electron. Lett. 29(18), 1614-1615 (1993). 